\title{
Heterogeneidad ambiental y alteraciones antrópicas en comunidades de manglar en el pacífico sur de México
}

\author{
Environmental heterogeneity and anthropic alterations in mangrove communities \\ in the Southern Pacific of Mexico
}

\section{Ameyali Moreno-Martínez ${ }^{1}$, Gustavo Álvarez-Arteaga ${ }^{2}$, María Estela Orozco-Hernández $^{3}$}

[Recibido: 24 de enero 2020, Aceptado: 26 de agosto 2020, Corregido: 1 de octubre 2020, Publicado: 1 de enero 2021]

\section{Resumen}

[Introducción]: La heterogeneidad ambiental de los manglares está consistentemente relacionada con factores biofísicos y antrópicos locales. Debido a que estos ecosistemas están sometidos a distintas presiones naturales o antrópicas, es necesario comprender el efecto de estas prácticas de manejo sobre sus componentes, para guiar acciones conducentes a incrementar su grado de resiliencia ante fenómenos meteorológicos extremos. [Objetivo]: La propuesta de esta investigación consistió en evaluar la condición ambiental de 4 comunidades de manglar a partir del estudio de los componentes suelo y vegetación, mediante un conjunto de propiedades edáficas y dasométricas, en la zona norte de la laguna de Coyuca, México. [Metodología]: La selección de los sitios de estudio y muestreo consideró su representatividad espacial, grado de alteración y proximidad a la línea de costa. Se caracterizó la estructura y biomasa arbórea y se tomaron muestras de suelo a una profundidad de $30 \mathrm{~cm}$ para su análisis en laboratorio. [Resultados]: El inventario forestal mostró la presencia de las especies Rhizophora mangle, Laguncularia racemosa y Conocarpus erectus, cuya distribución está relacionada, principalmente, con el régimen hídrico del suelo, pH y salinidad. El almacén de carbono aéreo y edáfico fluctuó entre 41.5 y $165 \mathrm{Mg} \mathrm{ha}^{-1}$, siendo consistente con su grado de perturbación. [Conclusiones]: Los principales efectos de las actividades antrópicas fueron la pérdida parcial de la estructura del bosque por tala y quema, compactación de la capa superficial del suelo, lo que deriva en el incremento de la densidad aparente y reducción del carbono almacenado en biomasa y suelo.

Palabras clave: Almacén de carbono; cambio climático; servicios ecosistémicos; suelos hidromórficos

\section{Abstract}

[Introduction] The environmental heterogeneity of mangroves is consistently related to local biophysical and anthropic factors. Because these ecosystems are subject to different natural or anthropic pressures, understanding the effect of these management practices on their components is necessary to take action to increase their degree of resilience to extreme weather events. [Objective] This research was aimed at evaluating the environmental condition

1 Estudiante de posgrado del Centro de Investigación y de Estudios Avanzados del Instituto Politécnico Nacional (CINVESTAV-IPN), Unidad Mérida, México; ameyalimorenomtz@gmail.com; https://orcid.org/0000-0001-9719-3627

2 Profesor investigador de la Facultad de Planeación Urbana y Regional (FaPUR), Universidad Autónoma del Estado de México, México; galvareza@uaemex.mx; https://orcid.org/0000-0002-0260-3484

3 Profesor investigador de la Facultad de Planeación Urbana y Regional (FaPUR), Universidad Autónoma del Estado de México, México; eorozcoh@gmail.com; https://orcid.org/0000-0003-4816-7742

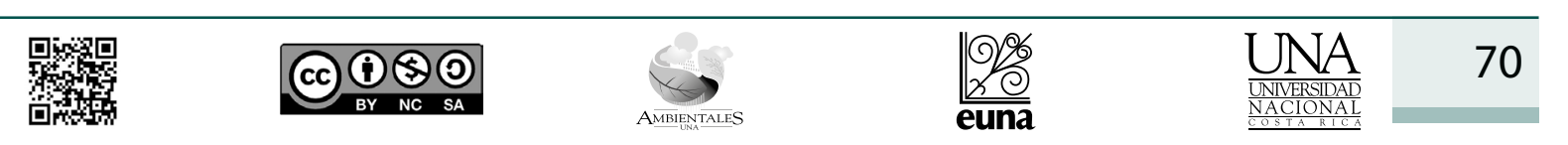




\section{Revista de CIENCIAS AMBIENTALES Tropical Journal of Environmental Sciences}

Revista de Ciencias Ambientales (Trop J Environ Sci) e-ISSN: 2215-3896 (Enero-Junio, 2021) . Vol 55(1): 70-85 DOI: https://doi.org/10.15359/rca.55-1.4

Open Access: www.revistas.una.ac.cr/ambientales e-mail: revista.ambientales@una.ac.cr

Moreno-Martínez A., Álvarez-Arteaga G., Orozco-Hernández M. E.

of 4 mangrove communities based on the study of soil and vegetation components, through a set of edaphic and dasometric properties, in the Northern area of the Coyuca Lagoon, Mexico. [Methodology] The selection of study and sampling sites considered their spatial representativeness, degree of alteration, and proximity to the coastline. Tree structure and biomass were characterized, and $30 \mathrm{~cm}$-deep soil samples were taken for lab analysis. [Results] Forest inventory showed the presence of the Rhizophora mangle, Laguncularia racemosa, and Conocarpus erectus species, whose distribution is mainly related to the soil hydric regimen, $\mathrm{pH}$, and salinity. Above-ground and soil carbon storage fluctuated between 41.5 and $165 \mathrm{Mg} \mathrm{ha}^{-1}$, being consistent with their degree of disturbance. [Conclusions] The main effects of the anthropic activities were partial loss of forest structure due to slash-and-burn, compaction of the topsoil, resulting in increased apparent density and reduced carbon stored in biomass and soil.

Keywords: Carbon store; climate change; ecosystem services; hydromorphic soils.

\section{Introducción}

La conservación de los manglares tiene una connotación que trasciende del contexto ambiental al socioeconómico, debido a que estos ecosistemas proporcionan múltiples servicios ecosistémicos como la captura de carbono (Donato et al., 2011; Mcleod et al., 2011), el reciclaje de nutrientes, su condición de hábitat para múltiples especies de peces, aves y crustáceos (Ellison, 2008), además de regular el ciclo hidrológico y ser fuente de alimento y empleo para los asentamientos humanos locales (Barbier et al., 2011). Pese a su importancia, estos ecosistemas están sujetos a presiones antrópicas que derivan en la disminución de su cobertura, biodiversidad y funciones ambientales, lo cual incrementa el riesgo y vulnerabilidad de las comunidades costeras ante fenómenos meteorológicos extremos (Gillanders et al., 2019) y contribuyen, adicionalmente, a la emisión de gases de efecto invernadero por su cambio de uso de suelo (Aburto-Oropeza et al., 2008; Mcleod et al., 2011; Rovai et al., 2018).

Los manglares son ecosistemas altamente variables en su composición, estructura y productividad, condición conferida por la heterogeneidad espaciotemporal de sus componentes bióticos y abióticos, como es el caso del suelo, la vegetación o los patrones hidrológicos locales (Feller et al., 2003; Hossain \& Nuruddin, 2016). En cuanto a los suelos de manglar, estos presentan rasgos característicos asociados a su régimen hídrico (fluctuaciones de la marea y aportes de agua continental), a la composición mineralógica de los sedimentos y a la cantidad y calidad de los restos orgánicos que ingresan al suelo (Otero et al., 2009). La interacción de dichos factores predispone la presencia de condiciones de saturación hídrica temporal o permanente, gradientes de salinidad, preservación de la materia orgánica en formas poco evolucionadas, así como la acumulación de sedimentos de composición variada, procedentes de la parte continental (Hossain \& Nuruddin, 2016). Cuando los manglares están sujetos a cambios de uso de suelo por actividades antrópicas como la agricultura y ganadería, es posible identificar diferentes patrones de alteración, identificados en la pérdida parcial o total de la estructura vegetal, reducción de los aportes orgánicos o alteraciones físicas y químicas de la capa superficial del suelo, procesos de degradación susceptibles de ser evaluados en fases iniciales o avanzadas con la finalidad de proponer acciones conducentes a su rehabilitación (Herrera-Silveira et al., 2012).

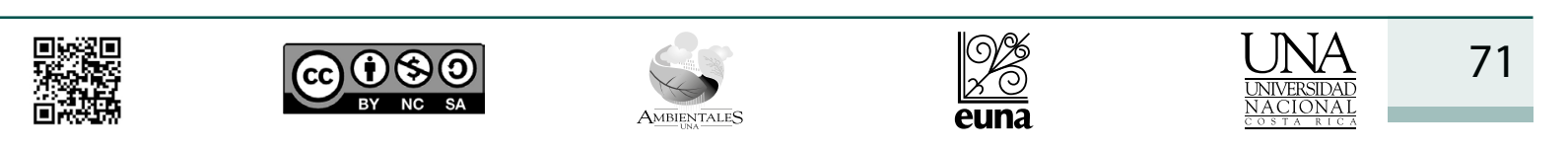




\section{Revista de CIENCIAS AMBIENTALES Tropical Journal of Environmental Sciences}

Revista de Ciencias Ambientales (Trop J Environ Sci) e-ISSN: $2215-3896$ (Enero-Junio, 2021) . Vol 55(1): 70-85 DOI: https://doi.org/10.15359/rca.55-1.4

Open Access: www.revistas.una.ac.cr/ambientales e-mail: revista.ambientales@una.ac.cr

Moreno-Martínez A., Álvarez-Arteaga G., Orozco-Hernández M. E.

Entre los parámetros fisicoquímicos del suelo que con mayor frecuencia se emplean como indicadores de su grado de conservación, resaltan aquellos que por su fácil obtención y sensibilidad a las alteraciones permiten detectar procesos incipientes de degradación (Karlen et al., 1997), por ejemplo: A) el color, relacionado indirectamente con la mineralogía del suelo, el contenido de materia orgánica, su fertilidad y los procesos anaeróbicos que tienen lugar en suelos hidromórficos (p. ej. suelos de manglar) (Campos \& Moreno-Casasola, 2009). B) El carbono orgánico del suelo (COS), relacionado con la disponibilidad y almacenamiento de carbono y otros nutrientes, altamente sensible a las prácticas de manejo a corto y mediano plazo; en tal sentido, Campos \& Moreno-Casasola (2009) y Kamrani et al. (2011) indican que la mineralización de la hojarasca en los suelos de manglar está fuertemente influenciada por su régimen hídrico, si el suelo se encuentra saturado de manera permanente o intermitente, la mineralización ocurre con menor rapidez debido a la ralentización de la actividad biológica, por lo que el $\mathrm{COS}$ tiende a preservarse en altas concentraciones, pero en formas poco transformadas. C) El $\mathrm{pH}$, indicador indirecto de la disponibilidad y movilidad de macro y micronutrientes, así como de elementos tóxicos para las plantas. Se ha documentado que los suelos de manglar poseen un amplio rango de condiciones, las cuales van de fuertemente ácidas a alcalinas (Hossain \& Nuruddin, 2016). D) La densidad aparente y el régimen hídrico, relacionados con la eficiencia de los procesos fisiológicos en las plantas (Herrera-Romero et al., 2019).

De acuerdo con estimaciones recientes, el Estado de Guerrero en México perdió aproximadamente el $18 \%$ de sus zonas de manglar entre 2010 y 2015 ; actualmente posee una cobertura de 6693 ha, de las cuales una cuarta parte se encuentra en algún proceso de degradación (Valderrama-Landeros et al., 2017). Entre las principales causas de perturbación identificadas para esta región se localizan el cambio de uso de suelo con fines agropecuarios y la implementación de desarrollos turísticos. Considerando que en estos ecosistemas la relación suelo-vegetación es fundamental para su funcionamiento, la propuesta de esta investigación consistió en evaluar la condición ambiental de 4 comunidades de manglar a partir del estudio de los componentes suelo y vegetación, mediante un cuadro de propiedades edáficas y dasométricas, dentro de la zona Norte de la Laguna de Coyuca, México.

\section{Metodología}

\subsection{Zona de estudio}

La investigación se llevó a cabo en el extremo norte de la Laguna de Coyuca (Figura 1), perteneciente al municipio de Coyuca de Benítez en el Estado de Guerrero, México. Se localiza aproximadamente a 32 kilómetros del Puerto de Acapulco, entre los límites 16 $6^{\circ} 53^{\prime} / 16^{\circ} 59^{\prime} \mathrm{N}$ y $99^{\circ} 58^{\prime} / 100^{\circ} 08^{\prime} \mathrm{O}$ (Tovilla-Hernández et al., 2009). El clima de la zona es tropical subhúmedo con lluvias en verano y sequías durante el invierno, la temperatura media anual es de $26{ }^{\circ} \mathrm{C}$ y la precipitación anual fluctúa entre los 900 y 1750 mm (García, 2004). Los suelos dominantes

\begin{tabular}{|c|c|c|}
\hline 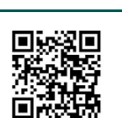 & (c) (i) (\$) () (2) & 72 \\
\hline
\end{tabular}




\section{Revista de CIENCIAS AMBIENTALES Tropical Journal of Environmental Sciences}

Revista de Ciencias Ambientales (Trop J Environ Sci) e-ISSN: 2215-3896 (Enero-Junio, 2021). Vol 55(1): 70-85 DOI: https://doi.org/10.15359/rca.55-1.4 Open Access: www.revistas.una.ac.cr/ambientales e-mail: revista.ambientales@una.ac.cr Moreno-Martínez A., Álvarez-Arteaga G., Orozco-Hernández M. E.

corresponden a Phaeozems, aunque es posible encontrar Gleysoles y Solonchaks en la zona de influencia de mareas (INEGI, 2013). La vegetación circundante a la laguna corresponde, principalmente, a comunidades de manglar, las cuales, en algunos casos, se encuentran impactadas por la influencia de asentamientos humanos dedicados a actividades primarias de autoconsumo o comercio local, como la agricultura, ganadería y extracción de madera (Valderrama-Landeros et al., 2017).

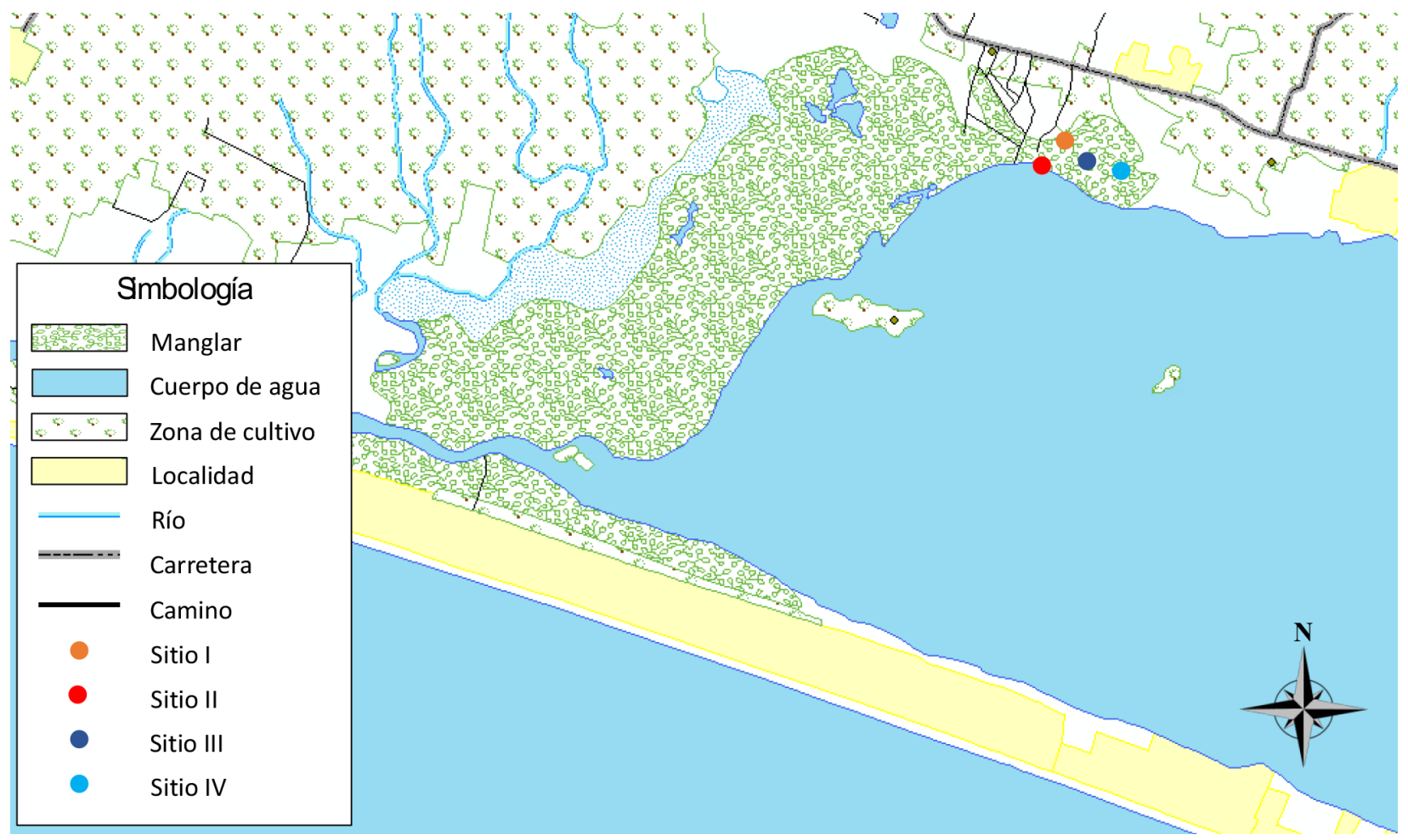

Figura 1. Ubicación de la zona de estudio y puntos de muestreos en zona de manglar de la Laguna de Coyuca. Figure 1. Location of the study area and sampling points in the mangrove area of Laguna de Coyuca.

\subsection{Selección de sitios y evaluación de la estructura y biomasa forestal}

Se realizó un análisis espacial mediante cartografía digital, posteriormente, un recorrido en la zona de estudio para identificar las comunidades de manglar próximas al borde de la laguna de Coyuca que han sido impactadas por actividades productivas. Se seleccionaron 4 sitios con una superficie de $125 \mathrm{~m}^{2}$ y en cada uno se realizó una caracterización ambiental general, para ello se considera su localización geográfica, distancia con respecto al borde de la laguna, presencia de estratos vegetales, indicios de alteración y régimen hídrico del suelo (Cuadro 1). Posteriormente se registró la especie, el diámetro a la altura del pecho (DAP $=1.3 \mathrm{~m}$ ) y área

\begin{tabular}{|c|c|c|}
\hline 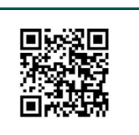 & (c) (i) () (2) & 73 \\
\hline
\end{tabular}




\section{Revista de
CIENCIAS AMBIENTALES Tropical Journal of Environmental Sciences}

Revista de Ciencias Ambientales (Trop J Environ Sci) e-ISSN: 2215-3896 (Enero-Junio, 2021) . Vol 55(1): 70-85 DOI: https://doi.org/10.15359/rca.55-1.4

Open Access: www.revistas.una.ac.cr/ambientales e-mail: revista.ambientales@una.ac.cr

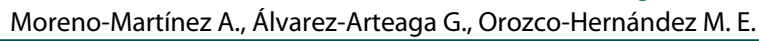

basal $(\mathrm{AB})$ de cada individuo con DAP superior a $2.5 \mathrm{~cm}$. A partir de estos datos se estimó la biomasa arbórea y la reserva de carbono con base en el protocolo propuesto por Kauffman et al. (2013), se hizo uso ecuaciones alométricas desarrolladas por Day et al. (1987) para especies de manglar.

Cuadro 1. Características generales de los sitios de manglar.

Table 1. General characteristics of mangrove sites.

\begin{tabular}{lllll}
\hline Sitio & Coordenadas (Norte / Oeste) & Altitud (msnm) & Estratos vegetales $^{\star}$ & Estresores $^{* *}$ \\
\hline 1 & $16^{\circ} 57^{\prime} 49^{\prime \prime} / 100^{\circ} 00^{\prime} 58^{\prime \prime}$ & 7 & $1,2,3$ & T, Q, RS \\
2 & $16^{\circ} 57^{\prime} 49^{\prime \prime} / 100^{\circ} 01^{\prime} 02^{\prime \prime}$ & 2 & $1,2,3$ & $\mathrm{~T}$ \\
3 & $16^{\circ} 57^{\prime} 51^{\prime \prime} / 100^{\circ} 00^{\prime} 59^{\prime \prime}$ & 8 & 1,2 & $\mathrm{~T}, \mathrm{RS}, \mathrm{C}, \mathrm{P}$ \\
4 & $16^{\circ} 57^{\prime} 46^{\prime \prime} / 100^{\circ} 00^{\prime} 54^{\prime \prime}$ & 11 & $1,2,3$ & $\mathrm{~T}$
\end{tabular}

${ }^{\star}$ 1=arbóreo; $2=$ arbustivo; $3=$ herbáceo. ${ }^{* *} \mathrm{~T}=$ tala; $\mathrm{Q}=$ quema; $\mathrm{RS}=$ residuos sólidos; $\mathrm{C}=$ compactación; $\mathrm{P}=$ pastoreo de ganado. ${ }^{* *}$ Los sitios se encuentran referidos según la Figura 1.

\subsection{Muestreo y evaluación de suelo}

Para el estudio de las propiedades fisicoquímicas y la estimación de la reserva de carbono en suelo a $30 \mathrm{~cm}$ de profundidad, se procedió a la toma de muestras en 4 puntos aleatorios dentro de la parcela de $125 \mathrm{~m}^{2}$, con 4 repeticiones por punto ( $\mathrm{n}=16$ por sitio). Adicionalmente, se tomó por triplicado una muestra de suelo inalterado mediante un cilindro de $100 \mathrm{~cm}^{3}$ para obtener la densidad aparente. Las muestras fueron llevadas al laboratorio para su preparación y análisis. En laboratorio se determinaron los siguientes parámetros fisicoquímicos de acuerdo con ISRIC (2002): textura por el método de Bouyoucos, $\mathrm{pH}$ con el método del potenciómetro, contenido de materia orgánica $(\mathrm{MO})$ por combustión en húmedo; densidad aparente (DA) por el método del cilindro de volumen conocido (Arshad et al., 1997); color del suelo mediante las tablas Munsell (1980); humedad a capacidad de campo (HCC) y a punto de marchitez permanente (HPMP) por el método propuesto por Ortiz (1996); conductividad eléctrica (CE) por medio de un conductímetro de campo en una relación suelo/agua (1:2.5). El carbono orgánico del suelo (COS) se estimó empleando la densidad aparente de cada muestra, multiplicada por el porcentaje de carbono y por la profundidad a la que fue tomada $(0.30 \mathrm{~m})$ (Ecuación 1) (González et al., 2008). Para aquellos datos con repeticiones se obtuvo la media aritmética con su desviación estándar.

$$
\mathrm{COS}=\mathrm{CO}^{\star} \mathrm{DA}^{\star} \mathrm{Ps}
$$

Donde:

COS = Carbono orgánico del suelo por superficie

$\mathrm{CO}=$ Carbono orgánico

$\mathrm{DA}=$ Densidad aparente

Ps $=$ profundidad del suelo

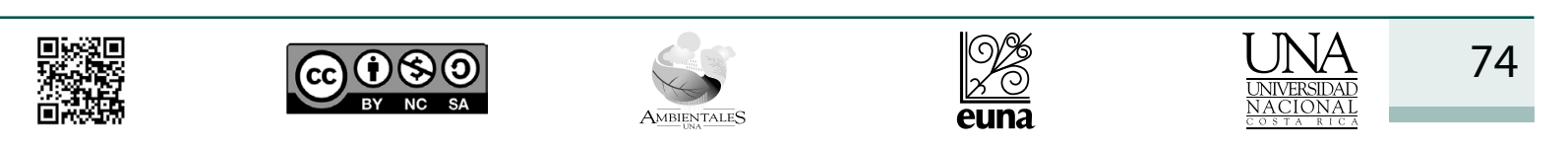




\section{Revista de CIENCIAS AMBIENTALES Tropical Journal of Environmental Sciences}

Revista de Ciencias Ambientales (Trop J Environ Sci) e-ISSN: 2215-3896 (Enero-Junio, 2021) . Vol 55(1): 70-85 DOI: https://doi.org/10.15359/rca.55-1.4 Open Access: www.revistas.una.ac.cr/ambientales e-mail: revista.ambientales@una.ac.cr Moreno-Martínez A., Álvarez-Arteaga G., Orozco-Hernández M. E.

\section{Resultados}

\subsection{Estructura y composición florística}

La información sobre la estructura y composición de las 4 comunidades de manglar procedentes de la zona norte de Laguna de Coyuca se describe en el Cuadro 2.

Cuadro 2. Características estructurales de las comunidades de manglar en la zona norte de la Laguna de Coyuca Table 2. Structural characteristics of mangrove communities in the north area of Laguna de Coyuca

\begin{tabular}{lllll}
\hline Sitio & Densidad & Especie dominante & Área basal $\left(\mathbf{m}^{2}\right)^{* *}$ & Área basal $\left(\mathbf{m}^{2}\right)^{* * *}$ \\
\hline I & 102 & $\begin{array}{l}\text { C. erectus } \\
\text { L. racemosa }\end{array}$ & 0.817 & 65.38 \\
II & 155 & $\begin{array}{l}\text { R. mangle } \\
\text { L. racemosa } \\
\text { L. racemosa }\end{array}$ & 1.09 & 87.08 \\
III & 27 & $\begin{array}{l}\text { L. racemosa } \\
\text { R. mangle }\end{array}$ & 0.76 & 106.88 \\
IV & 23 & una & 60.81 \\
\hline
\end{tabular}

${ }^{\star}$ Número de individuos registrados en una superficie de $125 \mathrm{~m}^{2} .{ }^{* \star}$ Área basal de los individuos registrados en una superficie de $125 \mathrm{~m}^{2} .{ }^{* *}$ Área basal de individuos extrapolados a una superficie de 1 ha.

Se identificó la dominancia de R. mangle en el sitio más próximo al borde de la laguna (Sitio II), con franjas de L. racemosa y C. erectus en transición hacia los puntos más alejados de la laguna (Sitios I, III y IV). Los sitios con mayor evidencia de perturbación (III y I) son aquellos con menor número de individuos (27 y 23, respectivamente). Sin embargo, en ambos sitos los individuos registrados son árboles adultos con DAP mayor a $30 \mathrm{~cm}$, los cuales no han sido removidos o talados, lo cual permite que su área basal sea superior al resto de los sitios (árboles con DAP entre 2.5 y $60 \mathrm{~cm}$ ).

\subsection{Caracterización fisicoquímica de los suelos}

A fin de establecer la relación entre la composición y estructura de la vegetación y el suelo, se realizó el análisis de un grupo de propiedades fisicoquímicas del suelo (Cuadro 3).

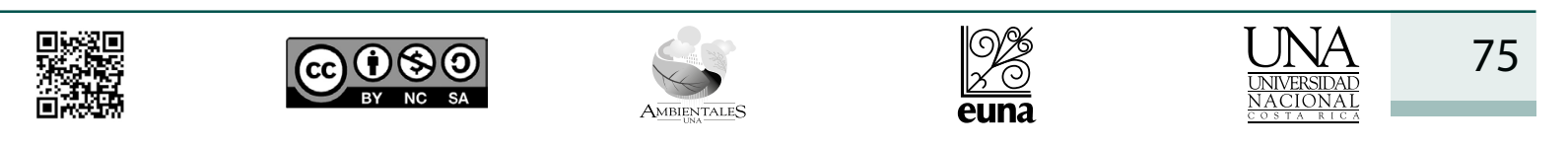




\section{Revista de
CIENCIAS AMBIENTALES Tropical Journal of Environmental Sciences}

Revista de Ciencias Ambientales (Trop J Environ Sci) e-ISSN: 2215-3896 (Enero-Junio, 2021). Vol 55(1): 70-85 DOI: https://doi.org/10.15359/rca.55-1.4 Open Access: www.revistas.una.ac.cr/ambientales e-mail: revista.ambientales@una.ac.cr Moreno-Martínez A., Álvarez-Arteaga G., Orozco-Hernández M. E.

Cuadro 3. Propiedades fisicoquímicas en suelos de manglar de la zona norte de la Laguna de Coyuca

Table 3. Physicochemical properties in mangrove soils in the north area of Laguna de Coyuca

\begin{tabular}{|c|c|c|c|c|c|c|c|c|c|c|}
\hline \multirow{2}{*}{$S$} & \multicolumn{2}{|l|}{ Color } & \multirow{2}{*}{$\mathrm{T}$} & \multirow{2}{*}{$\begin{array}{l}\text { DA } \\
\mathrm{g} \mathrm{cm}^{-3}\end{array}$} & \multirow{2}{*}{$\begin{array}{l}\mathrm{pH} \\
\mathrm{H}_{2} \mathrm{O}\end{array}$} & HCC & HРMP & \multirow{2}{*}{$\begin{array}{l}\text { COS } \\
\mathrm{g} \mathrm{kg}^{-1}\end{array}$} & \multirow{2}{*}{$\begin{array}{l}\mathrm{CE} \\
\mathrm{dS} \mathrm{m}^{-1}\end{array}$} & \multirow{2}{*}{$\begin{array}{l}\text { Salinidad } \\
\text { Ppm }\end{array}$} \\
\hline & Húmedo & Seco & & & & $\%$ & & & & \\
\hline I & $\begin{array}{l}\text { Gley } 1 \\
6 / 10 Y\end{array}$ & $\begin{array}{l}\text { Gley } 1 \\
4 / 10 Y\end{array}$ & CR & $\begin{array}{l}1.09 \\
(0.04)\end{array}$ & $\begin{array}{l}8.7 \\
(0.1)\end{array}$ & $\begin{array}{l}49.21 \\
(6.05)\end{array}$ & $\begin{array}{l}9.49 \\
(1.43)\end{array}$ & $\begin{array}{l}9.8 \\
(5.8)\end{array}$ & $\begin{array}{l}3.04 \\
(0.64)\end{array}$ & $1.80(0.44)$ \\
\hline II & 2.5YR2.5/1 & $10 \mathrm{YR} 4 / 2$ & $\mathrm{CR}$ & $\begin{array}{l}10.01 \\
(0.34)\end{array}$ & $\begin{array}{l}6.8 \\
(2.7)\end{array}$ & $\begin{array}{l}63.46 \\
(18.77)\end{array}$ & (7.77) & $(11.6)$ & $(1.38)$ & $2.48(0.83)$ \\
\hline III & $\begin{array}{l}\text { Gley } 1 \\
4 / 10 Y\end{array}$ & $10 \mathrm{YR} 6 / 2$ & CA & $\begin{array}{l}1.43 \\
(0.42)\end{array}$ & $\begin{array}{l}8.6 \\
(2.6)\end{array}$ & $\begin{array}{l}46.92 \\
(17.00)\end{array}$ & $\begin{array}{l}14.49 \\
(7.77)\end{array}$ & $\begin{array}{l}2.5 \\
(10.8)\end{array}$ & $\begin{array}{l}2.53 \\
(1.29) \\
3.67\end{array}$ & $1.19(0.80)$ \\
\hline IV & $2.5 \mathrm{Y} 3 / 1$ & $2.5 Y 5 / 2$ & $\mathrm{C}$ & $\begin{array}{l}1.07 \\
(0.39)\end{array}$ & $\begin{array}{l}7.6 \\
(2.5)\end{array}$ & $\begin{array}{l}54.91 \\
(17.04)\end{array}$ & $\begin{array}{l}19.84 \\
(8.51)\end{array}$ & $\begin{array}{l}19 \\
(11.6)\end{array}$ & $\begin{array}{l}3.6 / \\
(1.48)\end{array}$ & $1.80(0.77)$ \\
\hline
\end{tabular}

$\mathrm{S}=$ sitio; $\mathrm{T}=$ textura; $\mathrm{DA}=$ densidad aparente; $\mathrm{HCC}=$ humedad a capacidad de campo; $\mathrm{HPMP}=$ humedad a punto de marchitez permanente; $\mathrm{COS}=$ carbono orgánico del suelo; $\mathrm{CE}=$ conductividad eléctrica; $\mathrm{CR}=$ franco arcilloso; $\mathrm{CA}=-$ franco arenoso; $\mathrm{C}=$ franco. ${ }^{\star}$ Los valores dentro de los paréntesis indican la desviación estándar con respecto a la media.

\subsubsection{Propiedades físicas}

La clase textural de los suelos se clasifica dentro de los suelos francos con variantes que van de franco arcillosos (sitios I y II) a franco arenosos (sitio III). La presencia de tonalidades gleycas (grisáceas) en los sitios I y III denota la prevalencia de procesos hidromórficos en los suelos; en el caso de los sitios II y IV, los tonos pardos sugieren mejores condiciones de drenaje, un mayor contenido de materia orgánica y probablemente de nutrientes. La densidad aparente se situó en un rango de entre 1.01 y $1.43 \mathrm{~g} \mathrm{~cm}^{-3}$, donde el suelo del sitio III resultó ser el más compactado. El rango se humedad disponible en suelo se obtuvo a partir de la humedad a capacidad de campo y la humedad a punto de marchitez permanente, en tal sentido, el suelo con mayor capacidad de humedad disponible fue el sitio II (Figura 2). Esta condición puede estar relacionada con otros factores como la clase textural, la densidad aparente y el espacio poroso en suelo.

\begin{tabular}{|c|c|c|c|c|c|}
\hline 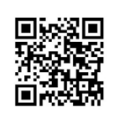 & (c) (1) (9) & $\underset{\text { AMBENTALES }}{\leftrightarrow}$ & $\frac{9 \%}{2 \%}$ & 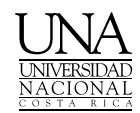 & 76 \\
\hline
\end{tabular}




\section{Revista de CIENCIAS AMBIENTALES Tropical Journal of Environmental Sciences}

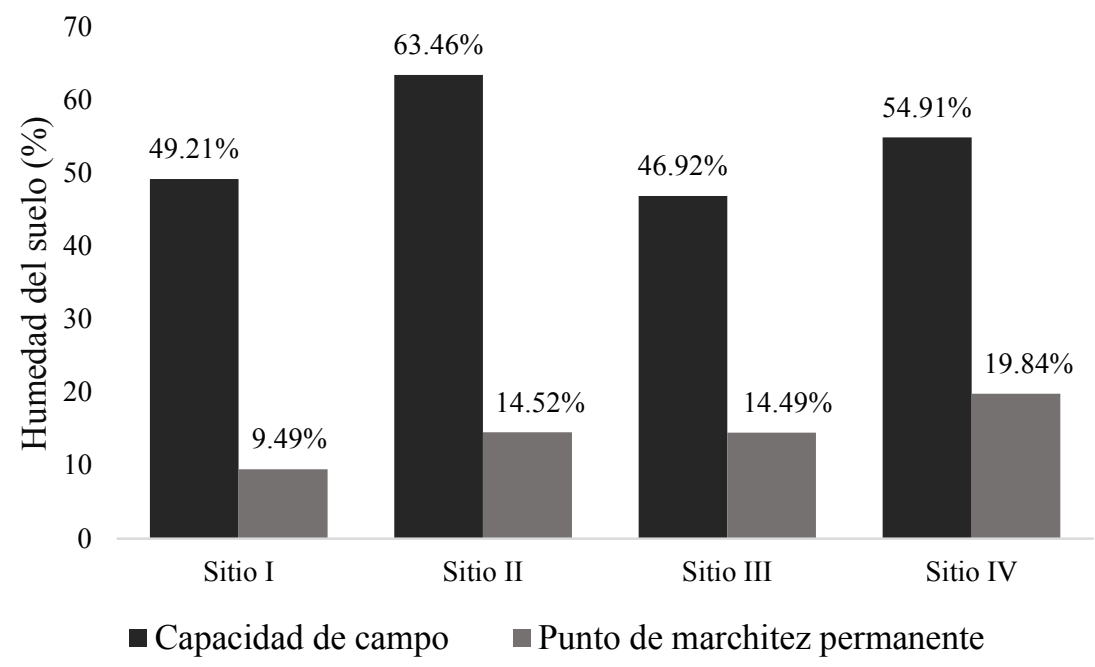

Figura 2. Humedad a capacidad de campo (CC) y humedad a punto de marchitez permanente (HPMP) para suelos de manglar en la zona norte de la Laguna de Coyuca.

Figure 2. Field capacity and permanent wilting point for mangrove soils in the north area of Laguna de Coyuca.

\subsubsection{Propiedades químicas}

El pH de los sitios de muestreo se situó en un rango de 6.8 (sitio II) a 8.7 (sitio I), esta variación se relaciona con la presencia de condiciones hidromórficas, dado que los suelos inundados (sitios I y III) son más alcalinos. El contenido de carbono orgánico del suelo se encontró en un rango de entre 2.5 (sitio III) a $22.6 \mathrm{~g} \mathrm{~kg}^{-1}$ (sitio II). Los valores de conductividad eléctrica en suelo se situaron entre 2.53 y $4.33 \mathrm{dS} \mathrm{m}^{-1}$, lo que denota una condición del suelo de ligera a moderadamente salina (DOF, 2002).

\subsection{Reservas de carbono}

Se obtuvo la reserva de carbono en biomasa arbórea y suelo de cada sitio de muestreo (Cuadro 4), a una profundidad de $30 \mathrm{~cm}$.

\begin{tabular}{|c|c|c|}
\hline 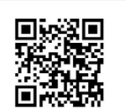 & (c) (1) (9) & 77 \\
\hline
\end{tabular}




\section{Revista de CIENCIAS AMBIENTALES Tropical Journal of Environmental Sciences}

Revista de Ciencias Ambientales (Trop J Environ Sci) e-ISSN: 2215-3896 (Enero-Junio, 2021). Vol 55(1): 70-85 DOI: https://doi.org/10.15359/rca.55-1.4

Open Access: www.revistas.una.ac.cr/ambientales e-mail: revista.ambientales@una.ac.cr Moreno-Martínez A., Álvarez-Arteaga G., Orozco-Hernández M. E.

Cuadro 4. Reserva de carbono en biomasa arbórea y suelos $\left(\mathrm{Mg} \mathrm{ha}^{-1}\right)$ de comunidades de manglar en la zona norte de la Laguna de Coyuca.

Table 4. Carbon pools in tree biomass and soils $\left(\mathrm{Mg} \mathrm{ha}^{-1}\right)$ of mangrove communities in the north area of Laguna de Coyuca.

\begin{tabular}{llllll}
\hline $\begin{array}{l}\text { Reservorio de } \\
\text { carbono }\end{array}$ & Sitio I & Sitio II & Sitio III & Sitio IV & Promedio \\
\hline Arbóreo & 142.01 & 141.63 & 284.03 & 150.79 & 179.25 \\
Suelo & $31.72(17.94)$ & $66.21(23.91)$ & $10.34(30.53)$ & $57.73(32.94)$ & 41.50 \\
Total & 173.73 & 207.84 & 294.37 & 208.52 & \\
\hline
\end{tabular}

* Los valores dentro de los paréntesis indican la desviación estándar con respecto a la media.

Los resultados sobre las reservas de carbono en biomasa arbórea fluctuaron entre 142 (sitio II) y $284 \mathrm{Mg} \mathrm{ha}^{-1}$, siendo el sitio III el que registró la mayor reserva. Por lo que concierne a la reserva de COS esta se situó entre los 10 (sitio III) y $66.2 \mathrm{Mg} \mathrm{ha}^{-1}$ (sitio II). El sitio con la mayor reserva de carbono en biomasa arbórea y en suelo fue el sitio III con $294.37 \mathrm{Mg} \mathrm{ha}^{-1}$.

\section{Discusión}

\subsection{Estructura y composición las comunidades de manglar}

Partiendo del análisis a escala de sitio, podemos establecer que las diferencias en la estructura y composición florística de las 4 comunidades estudiadas se relacionan, principalmente, con los cambios en la micro topografía, hidrología, condiciones del suelo (Herrera-Silveira et al., 2018). Como elemento adicional, las actividades antropogénicas tienden a alterar estos patrones de manera directa (p. ej. tala selectiva o desmonte total) o indirecta (p. ej. alteración de parámetros fisicoquímicos en agua y suelo). Por tanto, es evidente que la complejidad y respuesta de estas comunidades ante alteraciones naturales o antropogénicas no puede ser explicada por relaciones directas de causa-efecto, ya que obedecen a patrones multifactoriales más complejos.

Dentro de las 4 comunidades vegetales estudiadas se registró la presencia de 3 especies de mangle, previamente descritas para la zona de estudio por Castillo et al. (2018): Rhizophora mangle (mangle rojo), Laguncularia racemosa (mangle blanco) y Conocarpus erectus (mangle botoncillo); de acuerdo con la normatividad mexicana (DOF, 2010), dichas especies se encuentran dentro de la categoría de especies amenazadas. Por su distancia con respecto al borde de la laguna y posición fisiográfica, se corroboró que la distribución de las especies obedece a los patrones descritos por Day et al. (1987) y Romero-Berny \& Tovilla-Hernández (2009), en los que la zonificación de las especies está determinada en franjas. En este esquema, $R$. mangle predomina al borde de la laguna, con franjas de L. racemosa y C. erectus en transición hacia los puntos más alejados de la laguna (Figura 3).

\begin{tabular}{|c|c|c|c|c|c|}
\hline 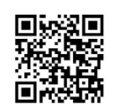 & (c) (1) () (2) & $\underset{\text { AMBENENALIES }}{\infty}$ & $\frac{1 \%}{\text { euna }}$ & 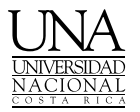 & 78 \\
\hline
\end{tabular}




\section{Revista de CIENCIAS AMBIENTALES Tropical Journal of Environmental Sciences}

Revista de Ciencias Ambientales (Trop J Environ Sci) e-ISSN: 2215-3896 (Enero-Junio, 2021) . Vol 55(1): 70-85 DOI: https://doi.org/10.15359/rca.55-1.4

Open Access: www.revistas.una.ac.cr/ambientales e-mail: revista.ambientales@una.ac.cr

Moreno-Martínez A., Álvarez-Arteaga G., Orozco-Hernández M. E.

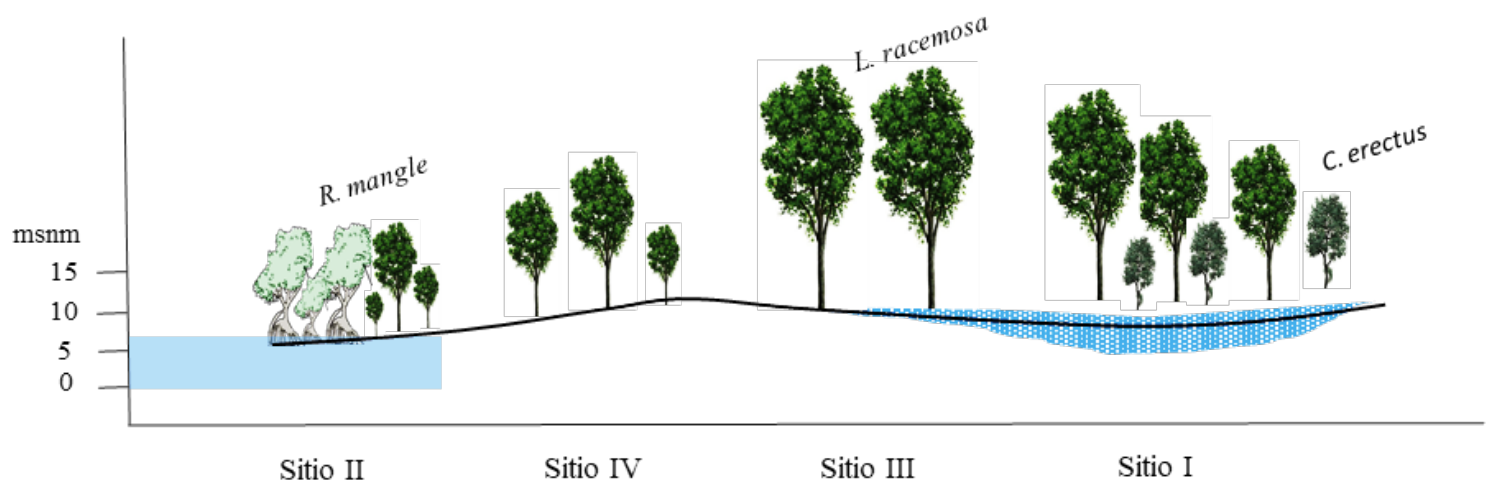

Figura 3. Distribución espacial de las especies de mangle en función del microrrelieve.

Figure 3. Spatial distribution of mangrove species as a function of microrelief.

\subsection{Características fisicoquímicas de los suelos}

$\mathrm{Si}$ bien, ha quedado establecido que, de manera general, los suelos de manglar comparten características propias; su heterogeneidad espaciotemporal y su relación con las comunidades vegetales ha sido objeto de múltiples investigaciones (Hossain \& Nuruddin, 2016). En el presente estudio, las variaciones de la clase textural de los suelos, que va de franco arcillosa a franco arenosa, sugieren que la variación granulometría de la fracción mineral se debe probablemente al arrastre y deposición de materiales en función de los flujos de agua y del microrrelieve (geoformas de crestas y depresiones), con lo cual, los sitios más deprimidos retienen partículas más finas, mientras que los sitios con mayor elevación contienen partículas más gruesas. Este supuesto concuerda con lo reportado por Herrera-Romero et al. (2019) para suelos costeros de Nayarit, México.

Dada la condición granulométrica intermedia dominante en los suelos estudiados, podría esperarse que los valores para la densidad aparente entre sitios no difieran significativamente; este supuesto tiene como excepción al sitio III, cuya densidad aparente es superior (30 \%) al resto de los sitios; tal condición puede ser atribuida al efecto que ocasiona la remoción parcial del estrato herbáceo y arbustivo, así como el paso frecuente de personas y ganado, lo que ha ocasionado la pérdida de estructura y sellamiento superficial favoreciendo la escorrentía y el arrastre de partículas finas, consistente con su clase textural (franco arenosa). Las condiciones reductomórficas denotadas por las tonalidades gleycas (grisáceas) de los suelos de los sitios I y III determinan restricciones en la disponibilidad de oxígeno y nutrientes requeridas para el establecimiento de determinadas especies herbáceas y arbustivas que acompañan al manglar (Ferreira et al., 2010).

Los valores de COS reportados en esta investigación $\left(2.5-22.6 \mathrm{~g} \mathrm{~kg}^{-1}\right)$ están dentro del segmento inferior del rango de estudios reportados por Hossain \& Nuruddin (2016) en suelos de

\begin{tabular}{|c|c|c|}
\hline 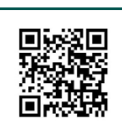 & (c) (1) () (2) & 79 \\
\hline
\end{tabular}




\section{Revista de CIENCIAS AMBIENTALES Tropical Journal of Environmental Sciences}

Revista de Ciencias Ambientales (Trop J Environ Sci) e-ISSN: 2215-3896 (Enero-Junio, 2021). Vol 55(1): 70-85 DOI: https://doi.org/10.15359/rca.55-1.4 Open Access: www.revistas.una.ac.cr/ambientales e-mail: revista.ambientales@una.ac.cr Moreno-Martínez A., Álvarez-Arteaga G., Orozco-Hernández M. E.

manglar en Asia, los cuales se sitúan entre 3.8 y $133.10 \mathrm{~g} \mathrm{~kg}^{-1}$, mientras que son similares a los obtenidos por Herrera-Romero et al. (2019) para sitios de marisma con inundación estacional en lagunas costeras en Nayarit. Tales contenidos de COS en la zona de estudio sugieren una reducida incorporación de restos orgánicas que derivan en una baja disponibilidad de nutrientes.

Al relacionar el contenido de materia orgánica en suelo con su densidad aparente, se observa una correlación inversa del $78 \%$, la cual es explicada por el efecto que tiene el aporte de materia orgánica sobre la fracción mineral, que incrementa el espacio poroso y disminuye la densidad del suelo, con lo que los sitios II y IV tendrían una mejor condición en relación a la disponibilidad de nutrientes y espacio poroso para que se lleve a cabo el intercambio hídrico y gaseoso entre suelo y plantas (Figura 4).

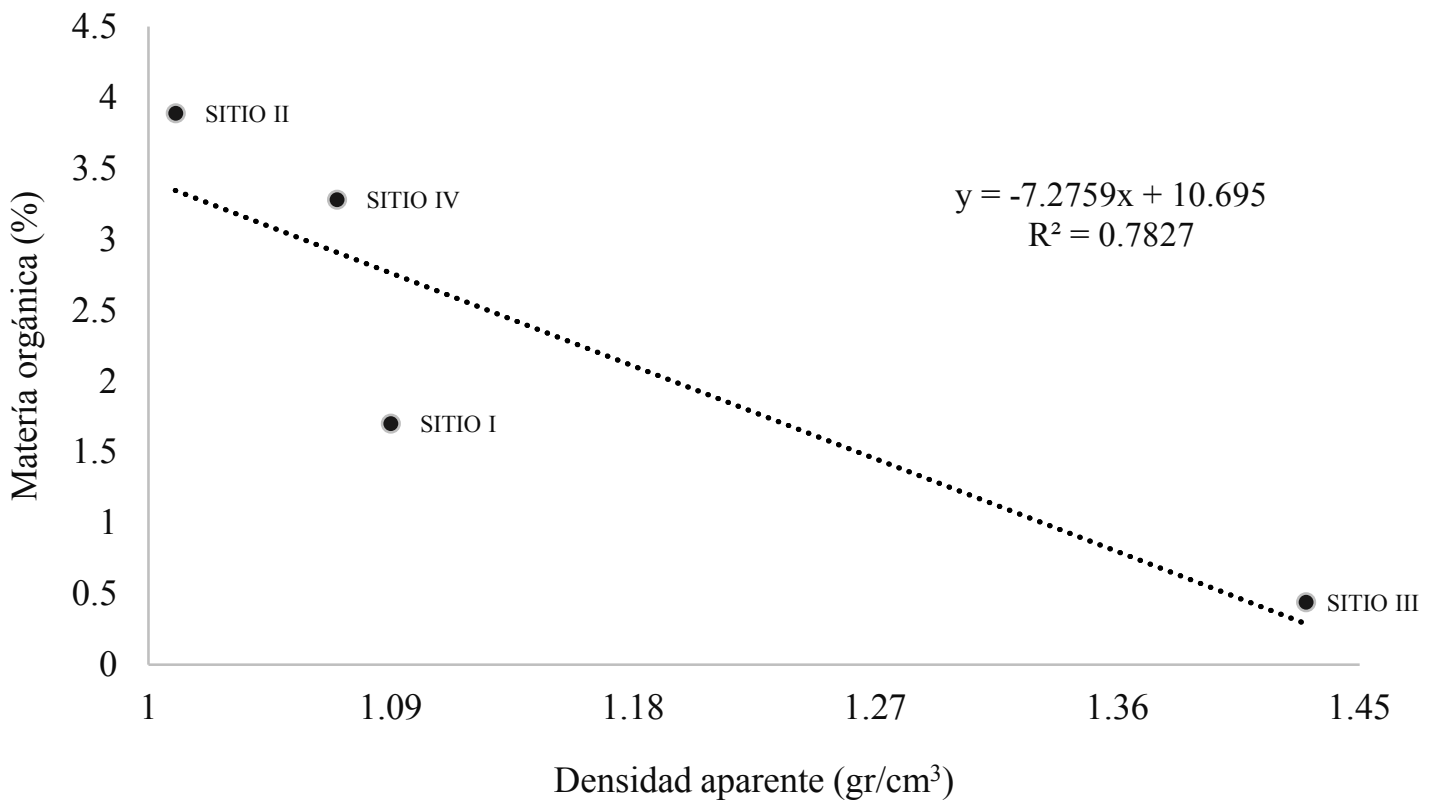

Figura 4. Relación entre el contenido de materia orgánica y la densidad aparente en suelos de manglar en la zona norte de la Laguna de Coyuca

Figure 4. Relationship between organic matter content and apparent density in mangrove soils in the north area of Laguna de Coyuca

\subsection{Reservas de carbono}

En el caso de México, la revisión realizada por Herrera-Silveira et al. (2018) establece que los manglares de la región Pacifico Sur (en la que se incluye la zona del presente estudio) almacenan un promedio de $810 \pm 323 \mathrm{Mg} \mathrm{Cha}^{-1}$, considerando los componentes vivos y muertos de la biomasa área y subterránea, y en el caso del suelo a una profundidad de $100 \mathrm{~cm}$. De manera

\begin{tabular}{|c|c|c|}
\hline 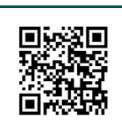 & (c) (i) () (2) & 80 \\
\hline
\end{tabular}




\section{Revista de CIENCIAS AMBIENTALES Tropical Journal of Environmental Sciences}

Revista de Ciencias Ambientales (Trop J Environ Sci) e-ISSN: $2215-3896$ (Enero-Junio, 2021) . Vol 55(1): 70-85 DOI: https://doi.org/10.15359/rca.55-1.4

Open Access: www.revistas.una.ac.cr/ambientales e-mail: revista.ambientales@una.ac.cr

Moreno-Martínez A., Álvarez-Arteaga G., Orozco-Hernández M. E.

comparativa, los datos obtenidos son consistentes con este estudio en la fracción de biomasa arbórea, ya que se ubican dentro del rango establecido por el equipo investigador para la misma región, correspondiente a $165 \pm 193 \mathrm{Mg} \mathrm{Cha}^{-1}$. A escala local podemos identificar un patrón que relaciona la menor reserva de COS con la presencia de condiciones hidromórficas y alcalinidad en los suelos (sitio I y III), que pudieran restringir la actividad biológica y la incorporación de materia orgánica al suelo. En contraparte, las mayores reservas de COS se tienen en sitios con un drenaje más eficiente y un $\mathrm{pH}$ ligeramente ácido.

\section{Conclusiones}

Las comunidades de manglar estudiadas presentan evidencia de heterogeneidad en cuanto a estructura y composición vegetativa y edáfica. Las evidencias sugieren que las condiciones como el microrrelieve, su posición con respecto a la cercanía de la laguna (gradiente de salinidad), así como las propiedades edáficas determinan las variaciones espaciales que restringen en mayor o menor medida los procesos fisiológicos de las especies vegetales, si bien estas condiciones son atribuibles a los procesos naturales y actividades antrópicas (p. ej. tala selectiva, pastoreo, actividades agrícolas) que alteran los procesos edáficos y contribuyen a la pérdida de biomasa.

Mediante los resultados obtenidos es posible establecer dos condiciones generales para los suelos: en el primer caso se incluyen a los suelos de los sitios I y III caracterizados por su régimen de saturación hídrica (suelos hidromórficos), condiciones alcalinas, bajos contenidos de materia orgánica, susceptibles a ser compactados cuando el contenido de arcillas domina sobre las otras fracciones minerales. El segundo caso correspondería a los suelos de los sitios II y IV, los cuales se caracterizan por baja saturación hídrica en los primeros $30 \mathrm{~cm}$ de profundidad, un $\mathrm{pH}$ cercano a neutro, mayor disponibilidad de materia orgánica, con salinidad ligeramente superior al primer grupo y una alta capacidad de retención de humedad disponible.

Los datos obtenidos en esta investigación permiten comprender la relación existente entre las condiciones edáficas y la estructura y composición florística de las comunidades de manglar estudiadas, así como mostrar los posibles efectos que pudieran tener dichas propiedades ante los impactos antrópicos, pudiendo ser elementos básicos para propiciar la recuperación de estos sistemas en función de la restitución de aquellas propiedades afectadas.

\section{6. Ética y conflicto de intereses}

Las personas autoras declaran que han cumplido totalmente todos los requisitos éticos y legales pertinentes, tanto durante el estudio como en la producción del manuscrito; que no hay conflicto de interés de ningún tipo; que todas las fuentes financieras se mencionan completa y claramente en la sección de agradecimientos, y que están totalmente de acuerdo con la versión final editada del artículo.

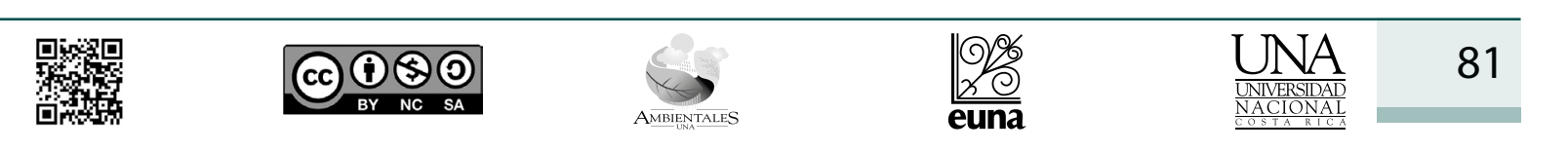




\section{Revista de
CIENCIAS AMBIENTALES Tropical Journal of Environmental Sciences}

Revista de Ciencias Ambientales (Trop J Environ Sci) e-ISSN: 2215-3896 (Enero-Junio, 2021). Vol 55(1): 70-85 DOI: https://doi.org/10.15359/rca.55-1.4

Open Access: www.revistas.una.ac.cr/ambientales e-mail: revista.ambientales@una.ac.cr Moreno-Martínez A., Álvarez-Arteaga G., Orozco-Hernández M. E.

\section{Agradecimientos}

Las personas autoras expresan su agradecimiento a la Universidad Autónoma del Estado de México por los apoyos recibidos para realizar esta investigación, así como a las personas anónimas que dictaminaron el escrito por sus aportes, los cuales enriquecieron el presente documento.

\section{Referencias}

Aburto-Oropeza, O., Ezcurra, E., Danemann, G., Valdez, V., Murray, J., \& Sala, E. (2008). Mangroves in the Gulf of California increase fishery yields. Proceedings of the National Academy of Sciences, 105(30), 10456-10459. https://doi.org/10.1073/pnas.0804601105

Arshad, M. A., Lowery, B., \& Grossman, B. (1997). Physical tests for monitoring soil quality. Methods for assessing soil quality, 49, 123-141. https://doi.org/10.2136/sssaspecpub49.c7

Barbier, E. B., Hacker, S. D., Kennedy, C., Koch, E. W., Stier, A. C., \& Silliman, B. R. (2011). The value of estuarine and coastal ecosystem services. Ecological monographs, 81(2), 169-193. https://doi.org/10.1890/10-1510.1

Campos, C. A., \& Moreno-Casasola, P. (2009). Suelos hidromórficos. En Costa sustentable, sustentabilidad de la costa veracruzana: Conservación y desarrollo. Breviario para describir, observar y manejar humedales (126-145). Instituto de Ecología AC, Semarnat.

Castillo, B., Gervasio, H., \& Bedolla, R. (2018). Estructura forestal de una zona de manglar en la laguna de Coyuca de Benítez, Guerrero. Revista Mexicana de Ciencias Forestales, 9(45), 6693. https://doi.org/10.29298/rmcf.v9i45.140

Day Jr, J. W., Conner, W. H., Ley-Lou, F., Day, R. H., \& Navarro, A. M. (1987). The productivity and composition of mangrove forests, Laguna de Terminos, Mexico. Aquatic Botany, 27(3), 267-284. https://doi.org/10.1016/0304-3770(87)90046-5

DOF. (2002). NOM-021-RECNAT-2000. Que establece las especificaciones de fertilidad, salinidad y clasificación de suelos. Diario Oficial de la Federación. México. D.F. 31 de diciembre de 2002.

DOF. (2010). NOM-059-SEMARNAT-2010. Protección ambiental-Especies nativas de México de flora y fauna silvestres-CAtegorías de riesgo y especificaciones para su inclusion, exclusion o cambio-Lista de especies en riesgo. Diario Oficial de la Federación. México D.F. 30 de diciembre de 2010.

Donato, D. C., Kauffman, J. B., Murdiyarso, D., Kurnianto, S., Stidham, M., \& Kanninen, M. (2011). Mangroves among the most carbon-rich forests in the tropics. Nature geoscience, 4(5), 293-297. https://doi.org/10.1038/ngeo1123

\begin{tabular}{|c|c|c|c|c|c|}
\hline 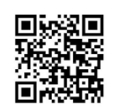 & (c) (1) () (2) & $\underset{\text { AMBENENALIES }}{\infty}$ & $\frac{1 \%}{\text { euna }}$ & 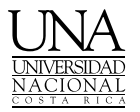 & 82 \\
\hline
\end{tabular}




\section{Revista de
CIENCIAS AMBIENTALES Tropical Journal of Environmental Sciences}

Revista de Ciencias Ambientales (Trop J Environ Sci) e-ISSN: 2215-3896 (Enero-Junio, 2021). Vol 55(1): 70-85 DOI: https://doi.org/10.15359/rca.55-1.4 Open Access: www.revistas.una.ac.cr/ambientales e-mail: revista.ambientales@una.ac.cr Moreno-Martínez A., Álvarez-Arteaga G., Orozco-Hernández M. E.

Ellison, A. M. (2008). Mangrove ecology-applications in forestry and coastal zone management. Aquatic botany, 89(2), 77. https://doi.org/10.1016/j.aquabot.2008.01.001

FAO (2017). Carbono orgánico del suelo. El potencial oculto. Organización de las Naciones Unidas para la Alimentación y la Agricultura. http://www.fao.org/3/b-i6937s.pdf

Feller, I. C., McKee, K. L., Whigham, D. F., \& O’neill, J. P. (2003). Nitrogen vs. phosphorus limitation across an ecotonal gradient in a mangrove forest. Biogeochemistry, 62(2), 145-175. https://doi.org/10.1023/A:1021166010892

Ferreira, T. O., Otero, X. L., de Souza Junior, V. S., Vidal-Torrado, P., Macías, F., \& Firme, L. P. (2010). Spatial patterns of soil attributes and components in a mangrove system in Southeast Brazil (São Paulo). Journal of Soils and Sediments, 10(6), 995-1006. https://doi.org/10.1007/ s11368-010-0224-4

García, E. (2004). Modificaciones al sistema de clasificación climática de köppen. Universidad Nacional Autónoma de México, Instituto de Geografía.

Gillanders, B. M., Fernandes, M., Gaylard, S., Sandhu, H., Waycott, M., Cavagnaro, T., \& Jones, A. R. (2019). Coastal carbon opportunities: summary report on the ecosystem services provided by blue carbon habitats in South Australia. Goyder Institute for Water Research Summary Report Series, (19/27), 3. http://www.goyderinstitute.org/_r2163/media/system/attrib/ file/607/Goyder_TRS-19-27\%20Coastal\%20C\%20Task3_summary\%20report_Final.pdf

González-Molina, L., Etchevers-Barra, J. D., \& Hidalgo-Moreno, C. (2008). Carbono en suelos de ladera: Factores que deben considerarse para determinar su cambio en el tiempo. Agrociencia, 42(7), 741-751. http://www.scielo.org.mx/scielo. php?pid=S1405-31952008000700001\&script=sci_arttext

Herrera-Romero, J. A., Bojórquez-Serrano, J. I., Can-Chulim, A., Madueño-Molina, A., \& García-Paredes, J. D. (2019). Salinidad y propiedades de suelos de las barras costeras en marismas nacionales de México. Revista Biociencias, 6. https://doi.org/10.15741/revbio.06.e412

Herrera-Silveira, J. A., Zaldivar-Jiménez, C. Teutli-Hernández, R. Pérez-Ceballos, J. Caamal, \& Andueza, T. (2012). Rehabilitación de manglares en el estado de Yucatán sometidos a diferentes condiciones hidrológicas y nivel de impacto: El caso de Celestún y Progreso. Centro de Investigación y de Estudios Avanzados. Unidad Mérida. Informe Final SNIB-CONABIO. Proyecto GH009. México, D.F.

Herrera-Silveira, J. A., Rico, A. C., Sosa, J. C., Castro, S. C., Ojeda, S. M. M., Ramírez, J. R. \& Angulo, J. E. E. (2018). Base de datos de almacenes de carbono en los manglares de México. Elementos para Políticas Públicas, 2(1), 33-44.

\begin{tabular}{|c|c|c|c|c|c|}
\hline 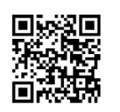 & (c) (1) () (2) & $\overbrace{\text { AMBENEMIIISS }}$ & $\frac{O \%}{20}$ & 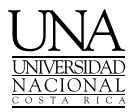 & 83 \\
\hline
\end{tabular}




\section{Revista de
CIENCIAS AMBIENTALES Tropical Journal of Environmental Sciences}

Revista de Ciencias Ambientales (Trop J Environ Sci) e-ISSN: 2215-3896 (Enero-Junio, 2021). Vol 55(1): 70-85 DOI: https://doi.org/10.15359/rca.55-1.4 Open Access: www.revistas.una.ac.cr/ambientales e-mail: revista.ambientales@una.ac.cr Moreno-Martínez A., Álvarez-Arteaga G., Orozco-Hernández M. E.

Hossain, M. D., \& Nuruddin, A. A. (2016). Soil and mangrove: a review. Journal of Environmental Science and Technology, 9(2), 198. https://doi.org/10.3923/jest.2016.198.207

INEGI. (2013). Metadatos de Edafología de México 1: 50 000, Serie II. Instituto Nacional de Estadística, Geografía e Informática, México.

ISRIC (2002). Procedures for soil analysis. International Soil Reference and Information Centre.

Kamrani, A., Jalili, A., Naqinezhad, A., Attar, F., Maassoumi, A., \& Shaw, S. (2011). Relationships between environmental variables and vegetation across mountain wetland sites, N. Iran. Biologia, 66(1), 76-87. https://doi.org/10.2478/s11756-010-0127-2

Karlen, D. L., Mausbach, M. J., Doran, J. W., Cline, R. G., Harris, R. F., \& Schuman, G. E. (1997). Soil quality: a concept, definition, and framework for evaluation (a guest editorial). Soil Science Society of America Journal, 61(1), 4-10. https://doi.org/10.2136/ sssaj1997.03615995006100010001x

Kauffman, J. B., Donato, D. C., \& Adame, M. F. (2013). Protocolo para la medición, monitoreo y reporte de la estructura, biomasa y reservas de carbono de los manglares (Documento de Trabajo 117). CIFOR.

Mcleod, E., Chmura, G. L., Bouillon, S., Salm, R., Björk, M., Duarte, C. M. \& Silliman, B. R. (2011). A blueprint for blue carbon: toward an improved understanding of the role of vegetated coastal habitats in sequestering $\mathrm{CO}_{2}$. Frontiers in Ecology and the Environment, 9(10), 552-560. https://doi.org/10.1890/110004

Munsell Color Co. (1980). Munsell Soil Color Charts. Baltimore, MD.

Ortiz Olguín, M. (1996). El muestreo de suelos salinos y sódicos. Universidad Autónoma de Chapingo.

Otero, X. L., Ferreira, T. O., Huerta-Díaz, M. A., Partiti, C. S. D. M., Souza Jr, V., Vidal-Torrado, P., \& Macías, F. (2009). Geochemistry of iron and manganese in soils and sediments of a mangrove system, Island of Pai Matos (Cananeia-SP, Brazil). Geoderma, 148(3-4), 318-335. https://doi.org/10.1016/j.geoderma.2008.10.016

Romero-Berny, E. I., \& Tovilla-Hernández, C. (2009). Estructura del manglar en el sistema lagunar costero de Carretas-Pereyra, reserva de la biósfera La Encrucijada, Chiapas, México. Lacandonia, Revista de Ciencias de la UNICACH, 3(1), 19-28.

Rovai, A. S., Twilley, R. R., Castañeda-Moya, E., Riul, P., Cifuentes-Jara, M., Manrow-Villalobos, M., \& Pagliosa, P. R. (2018). Global controls on carbon storage in mangrove soils. Nature Climate Change, 8(6), 534-538. https://doi.org/10.1038/s41558-018-0162-5

\begin{tabular}{|c|c|c|c|c|c|}
\hline 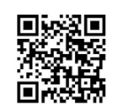 & (c) (i) () () & 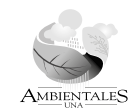 & $\frac{1 \%}{\text { euna }}$ & 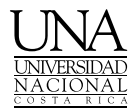 & 84 \\
\hline
\end{tabular}




\section{Revista de CIENCIAS AMBIENTALES \\ S Tropical Journal of Environmental Sciences}

Revista de Ciencias Ambientales (Trop J Environ Sci) e-ISSN: 2215-3896

(Enero-Junio, 2021) . Vol 55(1): 70-85 DOI: https://doi.org/10.15359/rca.55-1.4 Open Access: www.revistas.una.ac.cr/ambientales e-mail: revista.ambientales@una.ac.cr Moreno-Martínez A., Álvarez-Arteaga G., Orozco-Hernández M. E.

Rzedowski, J. (2006). Vegetación de México. (1ª ed.). Comisión Nacional para el Conocimiento y Uso de la Biodiversidad.

Tovilla-Hernández, C., Mora-Corro, S. A., Rojas-García, J., \& Vázquez-Lule, A. D. (2009). Caracterización del sitio de manglar Coyuca-Mitla. Comisión Nacional para el Conocimiento y Uso de la Biodiversidad http://www.conabio.gob.mx/conocimiento/manglares/doctos/caracterizacion/PS22_Coyuca_Mitla_caracterizacion.pdf

Valderrama-Landeros, L. H., Rodríguez-Zúñiga, M. T., Troche-Souza, C., Velázquez-Salazar, S., Villeda-Chávez, E., Alcántara-Maya, J. A., \& Ressl, R. (2017). Manglares de México: Actualización y exploración de los datos del sistema de monitoreo 1970/1980-2015. Comisión Nacional para el Conocimiento y Uso de la Biodiversidad. http://bioteca.biodiversidad.gob. $\mathrm{mx} /$ janium/Documentos/12889.pdf

\begin{tabular}{|c|c|c|c|c|c|}
\hline 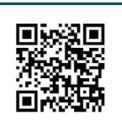 & (c) (i) () (2) & 8 & $\frac{1286}{20}$ & 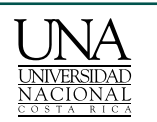 & 85 \\
\hline
\end{tabular}

